\title{
Surface Integrals and Monopole Charges in Non-Abelian Gauge Theories*
}

\author{
Clifford Henry Taubes $\dagger$ \\ Lyman Laboratory, Harvard University, Cambridge, MA 02138, USA
}

\begin{abstract}
We derive a formula which gives all the magnetic charges (topological invariants) of a monopole in the adjoint representation of a non-abelian gauge theory in terms of surface integrals at infinity.
\end{abstract}

\section{Introduction}

It has been known for some time that there exist topological invariants which are associated with static Yang-Mills-Higgs field configurations on Minkowski space [1-3]. In particular, suppose that the gauge group $G$ is a simple, compact Lie group. Further, assume that the Higgs field is in the adjoint representation of the Lie algebra $g$ of $G$. Every field configuration satisfying certain asymptotic conditions (c.f. Theorem 2.1) is known to define a gauge invariant set of integers $\left\{n_{a}\right\}_{a=1}^{\ell}, \ell \leqq$ rank $G[3]$. These integers are the aforementioned topological invariants. It is the purpose of this paper to prove that the integers $\left\{n_{a}\right\}_{a=1}^{\ell}$ are completely specified by surface integrals at $|x|=\infty$. For example, if $G=S U(n)$ and the representation of $g$ is the defining one, then

$$
Q_{k}=\lim _{R \rightarrow \infty} \frac{1}{4 \pi} \int_{|x|=R} \operatorname{tr}\left(\Phi^{k} F_{A}\right), \quad k \in\{1, \ldots, \ell\}
$$

completely determine the integers $\left\{n_{a}\right\}_{a=1}^{\ell}$. Here $\Phi$ is the Higgs field; the Lie algebra-valued two form $F_{A}$ is the curvature of the Yang-Mills connection $A$; and $x=\left(x^{1}, x^{2}, x^{3}\right)$ are cartesian coordinates on $\mathbb{R}^{3}$. For example, if $G=S U(2)$ then only $Q_{1}$ is needed. In this case the right hand side of (1.1) computes the winding number of the map

$$
\hat{\Phi}=\Phi /|\Phi|: S_{R}^{2}=\left\{x \in \mathbb{R}^{3}:|x|=R\right\} \rightarrow S^{2}=\{\sigma \in \operatorname{su}(2):|\sigma|=1\}[1]
$$

(see also [4], Proposition II.3.7.)

We remark that the right hand side of (1.1) is gauge invariant so it is not surprising that there should be some connection between the numbers $\left\{Q_{k}\right\}_{a=1}^{\ell}$ and the integer invariants $\left\{n_{k}\right\}_{k=1}^{\ell}$. This relationship is stated as Theorems $2.4-5$. The proofs are contained in Sect. 3-5.

\footnotetext{
* Supported in part by the National Science Foundation under Grant No. PHY 79-16812

$\dagger$ Junior Fellow, Harvard University Society of Fellows
} 


\section{The Topological Invariants}

We begin by establishing our notation. For convenience, we realize $G$ as an embedded submanifold in the vector space $\mathbb{M}(m ; \mathbb{C}$ or $\mathbb{R})$ of $m \times m$ matrices for some $m$. Let $\operatorname{tr}(\cdot)$ denote a normalized trace on $\mathbb{M}$. For $\sigma \in \mathbb{M}$,

$$
|\sigma| \equiv\left(\operatorname{tr}\left(\sigma^{\dagger} \sigma\right)\right)^{1 / 2}
$$

If $A$ is a connection on the principal $G$ bundle $\mathbb{R}^{3} \times G$ then the curvature of $A$ is the $g$ valued 2-form $F_{A}$ given by the well-known formula

$$
F_{A}=d A+A \wedge A \text {. }
$$

If $\Phi$ is a section of $\mathbb{R}^{3} \times g$ then $A$ defines a covariant derivative by

$$
D_{A} \Phi=\left(\nabla_{A} \Phi\right)_{i} d x^{i}=d \Phi+[A, \Phi] .
$$

We identify a gauge transformation with a map $g: \mathbb{R}^{3} \rightarrow G$ and $(A, \Phi)$ transforms under the action of $g$ in the usual way.

Let $\hat{x}$ denote a point on $S^{2}$ and suppose that

$$
\hat{\Phi}(\hat{x})=\lim _{t \rightarrow \infty} \Phi(t \hat{x})
$$

defines a map of $S^{2}$ into $g$. Let $h=\hat{\Phi}(1,0,0)$. Then define the Lie subgroup $J \subset G$ as

$$
J=\left\{g \in G: g h g^{-1}=h\right\} .
$$

Theorem 2.1. (Theorem II.3.1 of [4].) Let $A$ be a $C^{1}$ connection on $\mathbb{R}^{3} \times G$ and $\Phi a C^{1}$ section of $\mathbb{R}^{3} \times g$. Assume that

$$
\lim _{R \rightarrow \infty} \sup _{|x|=R}(1-|\Phi|)=0
$$

and that for some $\delta>0$,

Then

$$
|x|^{1+\delta}\left|\nabla_{A} \Phi\right| \leqq \text { const. }
$$

(a) There exists a gauge such that $\hat{\Phi}(x)$ is a continuous map from $S^{2}$ into $\mathrm{g}$.

(b) The configuration $(A, \Phi)$ defines a homotopy class $[(A, \Phi)] \in \Pi_{2}(G / J)$. (c.f. Proposition 2.2).

(c) The class $[(A . \Phi)]$ is invariant under $C^{1}$ gauge transformations.

(d) Suppose that $(a, \phi)$ is respectively a $C^{1}$ g-valued 1 -form and a $C^{1}$ section of $\mathbb{R}^{3} \times g$ which satisfy

$$
\lim _{R \rightarrow \infty} \sup _{|x|=R}|\Phi|=0=\lim _{R \rightarrow \infty} \sup _{|x|=R}|x||a|
$$

Then

$$
[(A+a, \Phi+\phi)]=[(A, \Phi)] .
$$

In order to be more specific, we note that the map

$$
\rho: G / J \rightarrow M=\left\{g h g^{-1}: g \in G\right\} \subset g
$$

is a diffeomorphism (c.f. [5], Theorem 2.9.4). 
Proposition 2.2. The map $\hat{\Phi}(\hat{x})$ takes values in $M$. Further, the map

is at least $C^{0}$.

$$
\hat{h}(\hat{x})=\rho^{-1}(\hat{\Phi}(\hat{x})): S^{2} \rightarrow G / J
$$

By definition, the class $[(A, \Phi)]=[\hat{h}]$.

We remark that Proposition 2.2 is a restatement of Lemmas II.4.3, 4 of [4]. However, there are some errata in the statement and proof of Lemma II.4.3 so we prove Proposition 2.2 in full in Sect. 4, c.f. Lemma 4.3.

The homotopy class $[(A, \Phi)] \in \Pi_{2}(G / J)$ is the topological invariant alluded to in the introduction. In order to categorize $[(A, \Phi)]$ we remark that the lie algebra $j$ of $J$ is necessarily the direct sum of a commuting algebra $h$ of dimension $\ell$ and a semi-simple lie algebra $g^{\prime}$ of $\operatorname{rank} s=\operatorname{rank} G-\ell$.

Theorem 2.3. The group $\Pi_{2}(G / J)$ is isomorphic to $\mathbb{Z}^{\ell}$.

Thus, if $(A, \Phi)$ satisfies $(2.5 \mathrm{a}, \mathrm{b})$, the topological invariant $[(A, \Phi)]$ is completely determined by a set of integers $\left\{n_{a}\right\}_{a=1}^{\ell}$.

Theorem 2.4. Let $G=S U(n) \subset \mathbb{M}(n, \mathbb{C})$. Suppose that $(A, \Phi)$ satisfies the conditions of Theorem 2.1 and in addition that $F_{A}$ and $D_{A} \Phi$ are square integrable. There exist constants $b_{a}^{k}$ with $a, k=1, \ldots, \ell$ which depend only on $A d_{G} h$ such that

$$
n_{a}=\sum_{k=1}^{\ell} b_{a}^{k} \lim _{R \rightarrow \infty} \int_{|x|=R} \operatorname{tr}\left(\Phi^{k} F_{A}\right), \quad a=1, \ldots, \ell
$$

are integers. Further, the set $\left\{n_{a}\right\}_{a=1}^{\ell}$ specify $[(A, \Phi)]$ in $\Pi_{2}(G / J)$.

Theorem 2.5. Let $G=S p(n) \subset \mathbb{M}(2 n, \mathbb{C}) \quad$ or $\quad G=S O(2 n) \subset \mathbb{M}(2 n, \mathbb{R})$ or $G=$ $S O(2 n+1) \subset \mathbb{M}(2 n+1, \mathbb{R})$. Suppose that $(A, \Phi)$ satisfies the conditions of Theorem 2.1 and in addition that $F_{A}$ and $D_{A} \Phi$ are square integrable. There exist constants $b_{a}^{k} ; a, k=1, \ldots, \ell$ which depend only on $A d_{G} h$ such that

$$
n_{a}=\sum_{k=1}^{\ell} b_{a}^{k} \lim _{R \rightarrow \infty} \int_{|x|=R} \operatorname{tr}\left(\Phi^{2 k-1} F_{A}\right)
$$

is an integer for each $a \in\{1, \ldots, \ell\}$. Further, the set $\left\{n_{a}\right\}_{a=1}^{\ell}$ specifies $[(A, \Phi)]$ in $\Pi^{2}(G / J)$.

There is a general formula to compute the integers $\left\{n_{a}\right\}_{a=1}^{\ell}$ which is valid for any Lie group and all faithful representations. This is the content of Proposition 4.5.

The proofs of Theorems $2.3-5$ comprise the next three sections. In Sect. 3 we review the relevant topology of $G / J$ and prove Theorem 2.3. In Sect. 4, it is proved that the integrands in $(2.9,10)$ are pull-backs of a linear combination of generators of $H_{D R}^{2}\left(H_{D R}^{*}\right.$ is the DeRham cohomology complex). This fact and Hurewicz isomorphism [6] allow us to prove the general result expressed in Proposition 4.5. Theorems 2.4-2.5 follow from Proposition 4.5 and properties of the matrix representation of the classical groups. This is explained in Sect. 5.

As a parenthetical remark, we point out that in theories with no Higgs self interactions, finite energy solutions to the classical equations of motion exist for all simple groups $G$, subgroups $J$ and integers $\left\{n_{a}\right\}_{a=1}^{\ell}$ if all the integers are the same sign (with respect to a specific choice of generators of $\Pi_{2}(G / J)[4,7]$. 


\section{Algebraic Topology on $G / J$}

This section is a review of the algebraic topology behind the computation of $\Pi_{2}(G / J)$. The important result is that the second homotopy group is isomorphic to the second cohomology group of the universal covering of $G / J$. The implications of this isomorphism are summarized in Proposition 3.2, and the second cohomology group of the universal cover is described in Proposition 3.3.

Let $\widetilde{G}$ be the universal covering space of $G$. There is a natural covering projection

$$
\tilde{G} \rightarrow G .
$$

Further, identifying $G / J$ with $M$ defined by (2.7) we have

$$
G / J=\left\{g h g^{-1}: g \in G\right\}=\left\{g h g^{-1} ; g \in \tilde{G}\right\} .
$$

Define the group $\tilde{J}$ by replacing $G$ by $\tilde{G}$ in equation (2.4). Then

$$
G / J=\tilde{G} / \tilde{J} .
$$

Let $\widetilde{J}_{(0)}$ be the component of $\widetilde{J}$ which is path connected to the identity. The projection

$$
p^{\prime}: \tilde{G} / \tilde{J}_{0} \rightarrow \tilde{G} / \tilde{J}=G / J
$$

is a covering space map. Since $\Pi_{1}(\widetilde{G})=\Pi_{0}\left(\widetilde{J}_{(0)}\right)=(0)$, the long exact homotopy sequence of fibration $\left[6\right.$, Theorem 7.2.10] implies that $\Pi_{1}\left(\tilde{G} / \widetilde{J}_{(0)}\right)=(0)$. Thus $\tilde{G} / \widetilde{J}_{(0)}$ is the universal covering space of $G / J$.

Let $x$ be the image of $J$ in $G / J$ and $x_{0}$ the image of $\tilde{J}_{(0)}$ in $\tilde{G} / \widetilde{J}_{(0)}$. The following proposition is standard [6, Theorem 2.4.5 and 7.2.10].

Proposition 3.1. Let $M$ be a simply connected space and $m \in M$. Suppose that $\psi$ maps the pair $(M, m)$ into $(G / J, x)$ continuously. Then there exists a unique lifting

$$
\tilde{\psi}:(M, m) \rightarrow\left(\widetilde{G} / \widetilde{J}_{(0)}, x_{0}\right)
$$

such that $p^{\prime} \psi=\psi$. In addition, the projection $p^{\prime}$ induces an isomorphism between $\Pi_{n}\left(\widetilde{G} / \widetilde{J}_{(0)}, x_{0}\right)$ and $\Pi_{n}(G / J, x)$ for all $n \geqq 2$.

Because $\Pi_{1}\left(\tilde{G} / \tilde{J}_{(0)}\right)=(0)$, the Hurewicz isomorphism is applicable, that is,

$$
H_{2}\left(\tilde{G} / \tilde{J}_{(0)} ; \mathbb{Z}\right) \approx \Pi_{2}\left(\tilde{G} / \tilde{J}_{(0)}\right) \text {. }
$$

Here $H_{*}(\cdot ; \mathbb{Z})$ are the singular homology groups with integral coefficients $[6$, Chapt. 7.5].

The group $J$ is a closed subgroup of $G$ and an analytic submanifold ([5], Lemma 2.9.2.) Since $\widetilde{G}$ is compact, $\widetilde{J}$ has a finite number of path components. Hence $\widetilde{G} / \widetilde{J}_{(0)}$ is a compact manifold. By poincaré duality [6, Theorem 6.2.18],

$$
\left.H_{2}\left(\tilde{G} / \tilde{J}_{(0)} ; \mathbb{Z}\right) \approx H^{m-2}\left(\tilde{G} / \tilde{J}_{(0)}\right) ; \mathbb{Z}\right)
$$

where $m=\operatorname{dim} \tilde{G} / \tilde{J}_{(0)}$ and $H^{*}(\cdot ; \mathbb{Z})$ are the singular cohomology groups with integral coefficients.

The Universal Coefficient Theorem [6] relates cohomology with integral coefficients to cohomology with real coefficients: 


$$
H^{m-2}\left(\tilde{G} / \tilde{J}_{(0)} ; \mathbb{Z}\right) \otimes \mathbb{R} \approx H^{m-2}\left(\tilde{G} / \tilde{J}_{(0)} ; \mathbb{R}\right) .
$$

A fundamental result of $R$. Bott [8] is that

$$
H^{m-2}\left(\widetilde{G} / \widetilde{J}_{(0)} ; \mathbb{Z}\right) \approx \mathbb{Z}^{\ell},
$$

where $\ell$ is the dimension of the center of $\tilde{J}_{(0)}$. This gives Theorem 2.3. In particular, $H^{m-2}$ is freely generated so

$$
H^{m-2}\left(\widetilde{G} / \tilde{J}_{(0)} ; \mathbb{R}\right) \approx \mathbb{R}^{\ell} .
$$

Finally, $H^{m-2}\left(\tilde{G} / \tilde{J}_{(0)} ; \mathbb{R}\right) \approx H_{D R}^{m-2}\left(\widetilde{G} / \widetilde{J}_{(0)}\right) \approx H_{D R}^{2}\left(\widetilde{G} / \widetilde{J}_{(0)}\right)$. Here $H_{D R}^{*}$ are the DeRham cohomology groups.

The above discussion is summarized in

Propositions 3.2. There are $\ell$ linearly independent generators $\left\{q_{1}, \ldots, q_{\ell}\right\}$ of $\Pi_{2}(\widetilde{G} / \widetilde{J})$ and $\ell$ linearly independent generators $\left\{\eta^{1}, \ldots, \eta^{\ell}\right\}$ of $H_{D R}^{2}\left(\widetilde{G} / \widetilde{J}_{(0)}\right)$. Any element $[\psi] \in \Pi_{2}(\widetilde{G} / \widetilde{J})$ has a unique expansion

$$
[\psi]=\sum_{a=1}^{\ell} n_{a} q_{a} \text { with } n_{a} \in \mathbb{Z} .
$$

There is an $\ell \times \ell$ matrix $\alpha_{a}^{k}$ which is independent of $[\psi]$ such that

$$
n_{a}=\sum_{k=1}^{\ell} \alpha_{a}^{k} \int_{s^{2}} \psi^{*}\left(\eta^{k}\right)
$$

Here $\psi \in[\psi]$ is any representative map.

With Eq. (3.11) in mind, we examine $H_{D R}^{2}\left(\widetilde{G} / \tilde{J}_{(0)}\right)$. With $\tilde{G} \subset \mathbb{M}(m)$ as a smooth submanifold, we represent $\widetilde{G} / \widetilde{J}_{(0)}$ as the quotient by matrix multiplication on the right:

$$
g \sim g^{\prime} \text { iff } g=g^{\prime} j \quad \text { with } j \in \tilde{J}_{0} .
$$

Let $\left\{x_{j}\right\}_{j=1}^{\operatorname{dim} g}$ be an orthonormal basis for $g$. That is

$$
\operatorname{tr}\left(x_{j}^{\dagger} x_{k}\right)=\delta_{j k} \text {. }
$$

The structure constants of $g,\left\{C_{i j}{ }^{k}\right\}$ are defined by the comutator

$$
\left[x_{i}, x_{j}\right]=c_{i j}{ }^{k} x_{k} \text {. }
$$

We denote a basis for the left invariant 1 -forms on $g$ by

$$
\omega^{j}=\operatorname{tr}\left(x_{j}^{\dagger} g^{-1} d g\right), \quad j=1, \ldots, \operatorname{dim} g .
$$

Here $d g=d g_{A B}$ is the restriction to $\widetilde{G}$ of the Euclidean one forms $d x_{A B}$ on $M$. The one forms $\omega^{j}$ satisfy the equations of Maurier-Cartan, namely

$$
d \omega^{k}=-\frac{1}{2} C_{i j}{ }^{k} \omega^{i} \wedge \omega^{j} .
$$

It is convenient to distinguish the elements of the basis $\left\{x_{j}\right\}$ which generate the subgroup $\tilde{J}_{(0)}$. Recall that the Lie algebra $j=h \oplus g^{\prime}$ where $[h, j]=0$. Let

$\left\{h_{a}\right\}_{a=1}^{\ell}$ be an orthonormal basis for $h$

$\left\{e_{\alpha}\right\}_{\alpha=1}^{\operatorname{dim} g^{\prime}}$ be an orthonormal basis for $g^{\prime}$ 
and $\left\{y_{A}\right\}_{A=1}^{\operatorname{dim} g-\operatorname{dim} g^{\prime}-\ell}$ be an orthonormal basis for the remaining generators of $g$. Then set

$$
\begin{array}{ll}
\omega^{a}=\operatorname{tr}\left(h_{a}^{\dagger} g^{-1} d g\right), & a=1, \ldots, \ell \\
\omega^{\alpha}=\operatorname{tr}\left(e_{\alpha}^{\dagger} g^{-1} d g\right), & \alpha=1, \ldots, \operatorname{dim} g^{\prime} \\
\omega^{A}=\operatorname{tr}\left(y_{A}^{\dagger} g^{-1} d g\right), & A=1, \ldots, \operatorname{dim} g-\operatorname{dim} g^{\prime}-\ell .
\end{array}
$$

Proposition 3.3. Let $p_{0}: \widetilde{G} \rightarrow \widetilde{G} / \widetilde{J}_{(0)}$ be the canonical projection. For each $a=1, \ldots, \ell$ there exists a closed two form $\eta^{a} \in \Lambda^{2}\left(\widetilde{G} / \widetilde{J}_{(0)}\right)$ such that

$$
p_{0}^{*} \eta^{a}=-\frac{1}{2} C^{a}{ }_{A B} \omega^{A} \wedge \omega^{B}
$$

Further the set $\left\{\eta^{a}\right\}_{a=1}^{\ell}$ generates $H_{D R}^{2}\left(\tilde{G} / \widetilde{J}_{(0)}\right)$ as an $\mathbb{R}$ module.

Proof. See, for example [9] and references therein.

\section{The Pull-Back of Cohomology from $\tilde{G} / \tilde{J}_{(0)}$}

We begin by studying the asymptotic behavior of $\Phi$ to demonstrate that for sufficiently large $r$, the Higgs field $\Phi(r \hat{x})$ maps $S^{2}$ into a tubular neighborhood of the manifold $M$ defined by (2.7). Hence we can compute the homotopy class defined by $\hat{\Phi}(\hat{x})$ from $\Phi(r x)$. The result is Proposition 4.5.

In this section it is always assumed that $(A, \Phi)$ satisfy the conditions of Theorem 2.1.

For $x \in \mathbb{R}^{3}$, define $\hat{x}=x /|x|$.

Lemma 4.1. There exists a smooth gauge such that

$$
\lim _{r \rightarrow \infty} \Phi(r \hat{x}) \equiv \hat{\Phi}(\hat{x})
$$

is a continuous map of $S^{2}$ into $g$. Further,

$$
\lim _{r \rightarrow \infty} r^{\delta}|\hat{\Phi}(\hat{x})-\Phi(r \hat{x})| \leqq \text { const. }
$$

Proof. It is always possible to choose a smooth gauge in which the radial component of the connection vanishes [10]. Then (4.1) follows from (2.5.6) by integration. The continuity of $\hat{\Phi}(\hat{x})$ is proved in Lemma II.4.1 of [4].

The statements above are gauge invariant under all gauge transformations $g(r \hat{x})$ which, as a function of $y=1 / r$, are Hölder continuous with exponent $\delta \leqq 1$ as $y \rightarrow 0$.

Let $\left\{t_{k}\right\}_{k=1}^{s}$ be an orthonormal basis for the Cartan sub-algebra $t \subset g$. Require that $t_{a}=h_{a}$ for $a \in\{1, \ldots, \ell\}$ where $\left\{h_{a}\right\}_{a=1}^{\ell}$ is defined in Sect. 3. By convention $h_{1} \equiv h$. It follows from (4.1) that there exists $R_{0}>0$ such that $|\Phi(x)| \geqq \frac{1}{2}$ if $|x|>R_{0}$. Set $v_{0}=(1,0,0)$ and $\bar{v}_{0}=(-1,0,0)$. For $R>R_{0}$ define

$$
V_{R}=\left\{x \in \mathbb{R}^{3}:|x|>R \text { and } \hat{x} \in S^{2} \backslash \bar{v}_{0}\right\} \text {. }
$$

Lemma 4.2. For some $R_{0}<R<\infty$ there exists $g \in C^{\infty}\left(V_{R}, G\right)$ and $\left\{\phi_{k}\right\}_{k=1}^{0} \in C^{\infty}\left(V_{R}\right)$ such that the following is true: For $x \in V_{R}$, 
(a) $\Phi(x)=\sum_{k=1}^{Ð} \phi_{k} g t_{k} g^{-1}$.

(b) $\lim r^{\delta}\left|\phi_{1}(r x)-1\right|<$ const.

(c) $\lim _{r \rightarrow \infty} r^{\delta}\left|\phi_{k}(r x)\right|<$ const. , for $k=2, \ldots, \sigma$.

Proof. Statement (a) follows from the fact that every element in $g$ is conjugate to an element in $t$. The smoothness conditions follow because $V_{R_{0}}$ is contractible and $|\Phi|>0$. Having established (a) we compute $D_{A} \Phi$ :

For $x \in V_{R_{0}}$,

$$
D_{A} \Phi=\sum_{k=1}^{\jmath} d \phi_{k} g t_{k} g^{-1}+\sum_{k=1}^{\doteq} \phi_{k}\left[A+d g g^{-1}, g t_{k} g^{-1}\right] .
$$

Using (4.3) and (2.5b) we obtain for $\hat{x} \in S^{2} \backslash \bar{v}_{0}, r_{1}>R_{0}$ and $k \in\{1, \ldots, o\}$ that

$$
\lim _{r \rightarrow \infty}\left|\phi_{k}(r \hat{x})-\phi_{k}\left(r_{1} \hat{x}\right)\right|<\text { const. } r_{1}^{-\delta} \text {. }
$$

Further, for $\hat{x}_{1}, \hat{x}_{2} \in S^{2} \backslash \bar{v}_{0}$, and $r>R_{0}$

$$
\lim _{r \rightarrow \infty} r^{\delta}\left|\phi_{k}\left(r \hat{x}_{1}\right)-\phi_{k}(r \hat{x})\right| \leqq \text { const. }
$$

The conclusion from (4.4) and (4.5) is that for each $k \in\{1, \ldots, \sigma\}$ there exists a constant $c_{k}$ such that

$$
\lim _{r \rightarrow \infty} r^{\delta}\left|\phi_{k}(r \hat{x})-c_{k}\right| \leqq \text { const. }
$$

By construction, $c_{k}=\delta_{k 1}$. Hence statements b) and c) of the Lemma.

Let

$$
e_{1}(x)=g(x) h_{1} g^{-1}(x) \in C^{\infty}\left(V_{R} ; M\right),
$$

where $g(x)$ is given in Lemma 4.2 and $M$ is defined in (2.7).

Lemma 4.3. The function $e_{1}(x)$ extends to an element in $C^{\infty}(\{x:|x|>R\} ; M)$. In addition,

(a) $\lim _{r \rightarrow \infty} r^{\delta}\left|\hat{\Phi}(\hat{x})-e_{1}(r \hat{x})\right| \leqq$ const.

(b) Proposition 2.2 is true.

(c) For all $r>R, \hat{\Phi}(\hat{x})$ and $e_{1}(r \hat{x})$ are homotopic as maps from $S^{2}$ into $M$.

Proof. The fact that $\phi_{1}(x)$ never vanishes in $\{x ;|x|>R\}$ implies that $e_{1}(x)$ has the stated extension. Statement (a) follows from statements (b, c) of Lemma 4.2 
and Eq. (4.1). Statement (a) of Lemma 4.3 implies that the sequence $\left\{e_{1}(m R \hat{x})\right\}_{m=1}^{\infty}$ is a Cauchy sequence in $M$ for fixed $\hat{x}$. Hence the limit, which is $\hat{\Phi}(\hat{x})$, takes values in $M$. This last fact and the previous Lemma imply (b). Statement (c) is implied by statement (a).

The orbit of each $h_{a}, a \in\{1, \ldots, \ell\}$, under $\operatorname{Ad}_{\tilde{G}}$ maps the group $\tilde{G}$ smoothly into its Lie algebra. Define $l_{a}: \widetilde{G} \rightarrow g$ by

$$
\ell_{a}(g)=g h_{a} g^{-1}
$$

We note that for each $a \in\{1, \ldots, \ell\}$, the map $\ell_{a}$ descends to a smooth map (which we also denote by $\ell_{a}$ ) of $\tilde{G} / \tilde{J}_{(0)}$ into $g$. This is because $\tilde{J}_{(0)}$ is generated by $\exp (j)$ and $[h, j]=0$.

Let $\hat{h}$ be the map defined in Proposition 2.2. Denote by $\tilde{h}: S^{2} \rightarrow \widetilde{G} / \widetilde{J}_{(0)}$ the unique lifting of $\hat{h}: S^{2} \rightarrow \tilde{G} / \tilde{J}$ as guaranteed by Proposition 3.1.

It follows from (c) of Lemma 4.3 that for $r>R$, the maps

$$
\hat{h}(r: \hat{x})=\rho^{-1}(e(r \hat{x})): S^{2} \rightarrow \tilde{G} / \tilde{J}
$$

are homotopic to $\hat{h}(\hat{x})$. In addition, the liftings of these maps,

$$
\tilde{h}(r ; \hat{x}): S^{2} \rightarrow \tilde{G} / \tilde{J}_{(0)}
$$

are homotopic to $\tilde{h}(\hat{x})$.

For $a \in\{1, \ldots, \ell\}$ define

$$
e_{a}(r ; \hat{x})=\ell_{a}(\tilde{h}(r, \hat{x}))
$$

Definition 4.4. For $r>R$ and $a \in\{1, \ldots, \ell\}$ define

$$
Q_{a}(r)=\int_{|x|=r} \operatorname{tr}\left(e_{a}(r ; \hat{x}) F_{A}\right)
$$

Proposition 4.5. Let $(A, \Phi)$ be as in Theorem 2.1. Then

$$
\int_{s^{2}} \tilde{h}^{*}\left(\eta^{a}\right)=\lim _{r \rightarrow \infty} Q_{a}(r)
$$

where the two cocycle $\eta^{a} \in H_{D R}^{2}\left(\tilde{G} / \tilde{J}_{(0)}\right)$ is defined in Proposition 3.3

Proof. By assumption, $A$ is $C^{1}$ so the integrand in (4.13) is smooth. Hence we have

$$
Q_{a}(r)=-\int_{|x|=r} \operatorname{tr}\left(D_{A} e_{a} \wedge A+A \wedge A\right),=-\int_{\substack{|x|=r \\ \hat{x} \in S^{2} \mid \bar{v}_{0}}} \operatorname{tr}\left(D_{A} e_{a} \wedge A+A \wedge A\right) .
$$

Recall that the set $V_{R}$ is contractible so that in $V_{R}, e_{1}(x)$ is given by (4.7) with $g(x) \in C^{\infty}\left(V_{R} ; \tilde{G}\right)$. Then up to an element of $\widetilde{J} / \tilde{J}_{0}$ (which is a discrete group)

$$
e_{a}(x)=g(x) h_{a} g^{-1}(x)
$$


An explicit computation of (4.15) gives

$$
\begin{aligned}
Q_{a}(r)= & -\int_{\substack{|x|=r \\
\hat{x} \in S^{2} \backslash \bar{v}_{0}}} \operatorname{tr}\left(g h_{a} g^{-1} g d g^{-1} \wedge g d g^{-1}\right) \\
& +\frac{1}{2} \int_{\substack{|x|=r \\
\hat{x} \in S^{2} \backslash \bar{v}_{0}}} \operatorname{tr}\left(\left[g h_{a} g^{-1}, A+g d g^{-1}\right] \wedge\left(A+g d g^{-1}\right)\right)
\end{aligned}
$$

Use (4.3) to estimate the second term in (4.17). That is, let $A_{L}$ be the component of $A+d g g^{-1}$ taking values in $g j g^{-1}$ and let $A_{T}$ be the orthogonal complement. Because $\phi_{1} \rightarrow 1$, equations (4.3) and (2.5b) imply that

$$
\left|\left[A+d g g^{-1}, g h_{a} g^{-1}\right]\right| \leqq \text { const. } r^{-1-\delta}
$$

and hence that

$$
\left|A_{T}\right|<\text { const. } r^{-1-\delta}
$$

Since the skew form

$$
g \rightarrow\left[g h_{a} g^{-1}, g\right] \quad(a \in\{1, \ldots, \ell\})
$$

has a kernal which includes $j$ and maps $j_{T}$ into itself, we conclude from (4.20) that

$$
\left|\operatorname{tr}\left(\left[g h_{a} g^{-1}, A_{T}\right] A_{T}\right)\right| \leqq \text { const. } r^{-2-2 \delta} .
$$

Therefore the second term in (4.17) is order $r^{-2 \delta}$. As for the first term in (4.17),

$$
\begin{aligned}
& -\int_{\substack{|x|=r \\
\hat{x} \in S^{2} \backslash \bar{v}_{0}}} \operatorname{tr}\left(g h_{a} g^{-1} g d g^{-1} \wedge g d g^{-1}\right)=\int_{\substack{|x|=r \\
\hat{x} \in S^{2} \backslash \bar{v}_{0}}} g^{*}\left(-\frac{1}{2} C^{a}{ }_{A B} \omega^{A} \wedge \omega^{B}\right), \\
& =\int_{\substack{|x|=r \\
\hat{x} \in S^{2} \mid \bar{v}_{0}}} g^{*} p_{(0)}^{*}\left(\eta^{a}\right)=\int_{\substack{|x|=r \\
\hat{x} \in S^{2} \backslash \bar{v}_{0}}} \tilde{h}(r ;)^{*}\left(\eta^{a}\right) .
\end{aligned}
$$

Because $\tilde{h}(r ; \cdot)$ is $C^{\infty}$ we can replace the point $\bar{v}_{0}$ in $(4.20)$. Since $\tilde{h}(r ; \cdot)$ is homotopic to $\tilde{h}$, Eq. (4.20) implies Eq. (4.14) and the Proposition is proved.

We now expand on the remarks in the opening paragraph of this section. The manifold $M$ is the orbit of $h$ under $\mathrm{Ad}_{G}$ and it is a regular, compact submanifold of $g \simeq \mathbb{R}^{\text {dim } g}$. As such, there exists a tubular neighborhood, $\mathcal{O}(M)$ of $M$ in $g$ and a $C^{\infty}$ projection $q: \mathcal{O} \rightarrow M$. (see [11], Chap. 12 for details.) We can specify $q$ uniquely by requiring that it be the orthogonal projection onto $M$ defined by the Killingform on $g$. This choice has the advantage that the projection commutes with the action of $\mathrm{Ad}_{G}$ on $M$ and $\mathcal{O}$.

Lemma's 4.1 and 4.3 imply that $\Phi(r \hat{x})$ maps $S^{2}$ into $\mathcal{O}$ for all $r$ larger than some 
$r_{1}$. It is not hard to show that

$$
(q \circ \Phi)(r \hat{x})=e_{1}(r \hat{x}) .
$$

We end this section with a summary:

Theorem 4.6. Let $(A, \Phi)$ satisfy the conditions of Theorem 2.1 Let $\rho: G / J \rightarrow M=$ $\mathrm{Ad}_{G} h$ be the canonical map. Let $q: \mathcal{O} \rightarrow M$ be a tubular neighborhood of $M$ in $g$ such that $q$ commutes with $\operatorname{Ad}_{G}$. Let $\ell_{a}: G \rightarrow g$ map $g \in G$ to $\operatorname{Ad}_{g} h_{a}$. There exist constants $b_{a}^{k} ; a, k \in\{1, \ldots, \ell\}$ which depend only on $\operatorname{Ad}_{G} h$ such that

$$
n_{a}=\sum_{k=1}^{\ell} b_{a}^{k} \lim _{r \rightarrow \infty} \int_{|x|=r} \operatorname{tr}\left(\ell_{a}\left(\widetilde{\rho-1 \circ q \circ \Phi) F_{A}}\right)\right.
$$

is an integer. The set $\left\{n_{a}\right\}_{a=1}^{\ell}$ specifies $[(A, \Phi)]$ in $\Pi^{2}(G / J)$. Here $\widetilde{\rho^{-1_{\circ}} q^{\circ} \Phi}$ is the lifting of $\rho^{-1} \circ{ }^{\circ} \Phi$ to $\tilde{G} / \tilde{J}_{(0)}$.

\section{The Classical Lie Groups}

Equation (4.21) can be simplified considerably if the properties of the specific matrix representation of a compact group $G$ are taken into account. A faithful, irreducible, unitary representation of $G$ of dimension $m$ is equivalent to an embedding of $G$ in $S U(m)$ or $S O(m)$ if the representation is real. Then $h$ is an $m \times m$ matrix and we can consider the matrix powers $h^{k}=h h \ldots h, k$ times. The set $\left\{h^{k}\right\}_{k=0}^{p-1}$ are linearly independent where $p$ is the number of distinct eigenvalues of $h$. (By construction, $h$ is traceless.) Let $\not h(h)$ be the linear span of $\left\{h^{k}\right\}_{k=0}^{p-1}$. Since $[\not h(h), j]=0$, the projection of $h(h)$ onto $g$ lies in the commuting subalgebra $h$ spanned by the orthonormal basis $\left\{h_{1}=h, h_{2} \ldots h_{\ell}\right\}$. The projection of $\not(h)$ on $g$ spans a subset $h^{\prime} \subseteq h$, say the subspace generated by $\left\{h_{1}, \ldots, h_{\ell^{\prime}}\right\}$ with $\ell^{\prime} \leqq \ell$. Then there exist constants $c_{a}^{k}$ where $a \in\left\{1, \ldots, \ell^{\prime}\right\}$ and $k \in\{1, \ldots, m\}$ such that

$$
h_{a}=\sum_{k=1}^{m} c_{a}^{k} h^{k}, \quad a=1, \ldots, \ell^{\prime} .
$$

Equation (5.1) suggests that

$$
\lim _{r \rightarrow \infty} \cdot \sum_{k=1}^{m} c_{a}^{k} \int_{|x|=r} \operatorname{tr}\left(\Phi^{k} F_{A}\right)=\int_{S^{2}} \tilde{h}^{*}\left(\eta^{a}\right),
$$

for $a \in\left\{1, \ldots, \ell^{\prime}\right\}$, and where $\eta^{a}$ is a generator of $H_{D \cdot R}^{2} \cdot\left(\tilde{G} / \tilde{J}_{(0)}\right)$ as discussed in Sect. 3 (Proposition 3.3.) In fact this is the case with a proviso, namely $F_{A}$ and $D_{A} \Phi$ are squared integrable.

Proposition 5.1. Let $(A, \Phi)$ satisfy the conditions of Theorem 2.1 and in addition suppose that $F_{A}$ and $D_{A} \Phi$ are square integrable. Let a faithful, irreducible representation of $G$ be given by an embedding of $G \subset S U(m)$ or $S O(m)$ for some $m$ such that (5.1)holds. Then (5.2) holds. 
Proof. Equation (5.2) holds with $\Phi$ replaced by $e_{1}(r \hat{x})$ as defined in (4.7). Lemma 4.2 and Eq. (5.3) imply that there exists $r_{1}>0$ such that $D_{A} e_{1} \in L_{2}\left(\mathbb{R}^{3} \backslash\left\{|x|<r_{1}\right\}\right)$. Using Kato's inequality (c.f. [4], Chap. VI.) we have $\nabla\left|\Phi-e_{1}\right| \in L_{2}\left(\mathbb{R}^{3} \backslash\left\{|x|<r_{1}\right\}\right)$ where $\nabla$ is the ordinary derivative. Lemma's 4.1 and 4.3 imply that $\left|\Phi-e_{1}\right| \in$ $L_{q}\left(\mathbb{R}^{3} \backslash\left\{|x|<r_{1}\right\}\right)$ for all $q>3 \delta^{-1}$. Then the Sobolev lemma (see e.g. [4], Chap. VI.) gives:

$$
\left|\Phi-e_{1}\right| \in L_{6}\left(\mathbb{R}^{3} \backslash\left\{|x|<r_{1}\right\}\right) .
$$

Fix once and for all a function $\beta(x) \in C_{0}^{\infty}\left(\mathbb{R}^{3}\right)$ with the property that

(a) $0 \leqq \beta(x) \leqq 1$,

(b) $\quad \beta(x)=1 \quad$ if $|x| \leqq 3 / 4$,

(c) $\quad \beta(x)=0 \quad$ if $|x| \geqq 1$.

Set $\beta_{r}(x)=\beta(x / r)$ which we define for $r>0$. Then for $r>2 r_{1}$,

$$
\begin{aligned}
\Delta(r)= & \left|\int_{|x|=r} \operatorname{tr}\left(\Phi^{k} F_{A}\right)-\int_{|x|=r} \operatorname{tr}\left(e_{1}^{k} F_{A}\right)\right| \\
\leqq & k \int_{|x| \leqq r}\left(1-\beta_{r}\right)\left(|\Phi|^{k-1}\left|D_{A} \Phi\right|\left|F_{A}\right|+\left|D_{A} e\right|\left|F_{A}\right|\right) \\
& +\int_{|x| \leqq r}\left|d \beta_{r}\right|\left(1-\beta_{r / 2}\right)|\Phi-e| k\left(|\Phi|^{k-1}+1\right)\left|F_{A}\right| .
\end{aligned}
$$

Here we have used Stoke's theorem along with the identities

$$
d \beta_{r}=d \beta_{r}\left(1-\beta_{r / 2}\right)
$$

and

$$
\Phi^{k}-e^{k}=\frac{1}{2}(\Phi-e)\left(\sum_{j=0}^{k-1} \Phi^{j} e^{k-1-j}\right)+\frac{1}{2}\left(\sum_{j=0}^{k-1} \Phi^{j} e^{k-1-j}\right)(\Phi-e) .
$$

Now using Hölder's inequality, we obtain

$$
\begin{aligned}
& \Delta(r) \leqq\left\|\left(1-\beta_{r / 2}\right) F_{A}\right\|_{L_{2}}\left(\left\|D_{A} \Phi\right\|_{L_{2}}+\left\|\left(1-\beta_{r / 2}\right) D_{A} e\right\|_{L_{2}}\right. \\
& \left.\left\|\nabla \beta_{r}\right\|_{L_{3}}\|\Phi-e\|_{L_{6}}\right) k\left(\|\Phi\|_{L_{\infty}}^{k-1}+1\right) .
\end{aligned}
$$

By scaling, $\left\|\nabla \beta_{r}\right\|_{L_{3}}=\left\|\nabla \beta_{1}\right\|_{L_{3}}$ so we conclude from (5.7) that

$$
\lim _{r \rightarrow \infty} \Delta(r)=0 \text {. }
$$

This proves Proposition 5.1.

Proof of Theorem 2.4 Let $G=S U(n)$ acting on $\mathbb{C}^{n}$. The number of different eigenvalues of $h$ is precisely the dimensions of $h$. Hence the projections of the powers $\left\{h^{k}\right\}_{k=1}^{n-\ell}$ onto $h$ span $h$. Thus Theorem 2.4 follows from Proposition 5.1.

Proof of Theorem 2.5. Case $1 . G=S p(n)$ acting on $\mathbb{C}^{2 n}$. An arbitrary element in the Lie algebra $s p(n)$ has the form [12, Sect. 65]

$$
A \otimes 1+S_{j} \otimes \tau_{j}
$$


where $\left(1, \tau_{j}\right)$ are unit quaternious; $A$ and $S_{j}$ are real $n \times n$ matrices but $A^{T}=-A$ while $S_{j}^{T}=S_{j}$. The Cartan subalgebra may be chosen to have the form

$$
t=\left\{D \otimes \tau_{3} ; D \text { a diagonal } n \times n \text { real matrix }\right\} .
$$

The eigenvalues of $h \in t$ come in \pm pairs since $\tau_{3}$ has eigenvalues $\pm i$. Hence we need only consider the odd powers of $h$ (the even powers are orthogonal to $s p(n)$.) We write

$$
h=H \otimes \tau_{3} \quad \text { with } H \text { diagonal. }
$$

It is not hard to see that the number of distinct eigenvalues of $h$ (the number of distinct eigenvalues of $H$ up to sign) is precisely the dimensions of $h$. Hence $h_{\text {odd }}(h)$ spans $h$. Case 1 follows now from Proposition 5.1.

Case 2. $G=S O(2 n)$ acting on $\mathbb{R}^{2 n}$. The Lie algebra so $(2 n)$ is the space of $2 n \times 2 n$ real skew-symmetric matrices. Let $\alpha, \beta, \sigma$ be $2 \times 2$ matrices defined by

$$
\begin{aligned}
& \alpha=\left(\begin{array}{rr}
1 & 0 \\
0 & -1
\end{array}\right), \\
& \beta=\left(\begin{array}{lr}
0 & 1 \\
1 & 0
\end{array}\right), \\
& \sigma=\left(\begin{array}{rr}
0 & 1 \\
-1 & 0
\end{array}\right) .
\end{aligned}
$$

Every element in so $(2 n)$ has the form [12, Sect. 65]

$$
A_{0} \otimes 1+A_{\alpha} \otimes \alpha+A_{\beta} \otimes \beta+S_{\sigma} \otimes \sigma
$$

where $A_{0}, A_{\alpha}, A_{\beta}$ and $S_{\sigma}$ are real $n \times n$ matrices with $A_{0, \alpha, \beta}$ antisymmetric but $S_{\sigma}$ symmetric. An element $h$ in the Cartan subalgebra can be put in the form

$$
h=H \otimes \sigma \quad \text { with } H \text { a diagonal } n \times n \text { matrix }
$$

Hence the eigenvalues come in \pm pairs also and we need only consider the odd powers of $h$. Once again the number of distinct eigenvalues of $h$ is precisely the dimension of $h$ and $h_{\text {odd }}(h)$ spans $h$. Case 2 follows from Proposition 5.1. Case 3. $G=S O(2 n+1)$ acting on $\mathbb{R}^{2 n+1}$. The group $S O(2 n+1)$ has rank $n$. The Lie algebra, $s o(2 n+1)$ is the space of $(2 n \times 1) \times(2 n+1)$ real skew-symmetric metrices. Every element in so $(2 n+1)$ has the form

$$
\left(\begin{array}{l|l}
s o(2 n) & 0 \\
\hline 0 \ldots & 0
\end{array}\right)+\left(\begin{array}{c}
0 \mid v \\
\hline-v \mid 0
\end{array}\right)
$$

where $v$ is an $n \times 1$ matrix. An element $h$ in the Cartan subalgebra may be taken to have the form

$$
h=\left(\begin{array}{c|c}
H \otimes \sigma & 0 \\
\hline 0 & 0
\end{array}\right)
$$

where $H$ is diagonal [12]. This case reduces to the case of $S O(2 n)$. 
Acknowledgement. The author warmly thanks Professors A. Jaffe and R. Bott for their many insights and suggestions. Thanks also to D. Groisser.

\section{References}

1. Coleman, S. : Classical lumps and their quantum descendents. In : New Phenomena and subnuclear Physics. Zichichi, A. (ed.) New York: Plenum Press 1977

2. Englert, F., Windey, P. : Phys. Rev. D14, 2728 (1976)

3. Goddard, P., Nuyts, J., Olive, D. : Nucl. Phys. B125, 1 (1977)

4. Jaffe, A., Taubes, C. : Vortices and monopoles. Boston : Birkhäuser 1980

5. Varadarajan, V.: Lie groups, lie algebras, and their representations. Englewood Cliffs, NJ : Prentice-Hall Inc. 1974

6. Spanier, E. : Algebraic Topology, New York : McGraw-Hill 1966

7. Taubes, C. : The existence of multimonopole solutions to the non-abelian Yang-Mills equations for arbitrary simple groups. Commun, Math. Phys. 80, 343-367 (1981)

8. Bott, R. : The geometry and representation theory of compact Lie groups. In : Representation Theory of Lie Groups. London Math. Soc. Lect. Note 34, Cambridge: Cambridge University Press 1979

9. Samelson, H. : Bull. Am. Math. Soc. 58, 2 (1952)

10. Uhlenbeck, K. : Removable singularities in Yang-Mills theory. Commun, Math. Phys (to appear)

11. Palais, R. : Foundations of global nonlinear analysis New York: W. A. Benjamin, Inc. 1968

12. Pontryagin, L. : Topological groups. New York: Gordon and Breach 1966

Communicated by A. Jaffe

Received November 14, 1980; in revised form November 26, 1980 
